\title{
A comparative study of protective mechanisms of glycine and L-arginine against cisplatin-induced nephrotoxicity in rat renal cortical slices
}

\author{
Yasmen YFK Mahran, Amani E Khalifa, Ebtehal El-Demerdash* \\ Pharmacology \& Toxicology Department, Faculty of Pharmacy, Ain Shams University, Cairo, Egypt.
}

\begin{abstract}
Amino acids exert nephroprotective effects in various forms of acute renal injury depending on their renal hemodynamic effects. The present study was designed to elucidate and compare the role of non hemodynamic mechanisms in protective actions afforded by glycine and $\mathrm{L}$-arginine against cisplatin (CDDP)-induced nephrotoxicity using rat renal cortical slices (RCS). We have investigated the possible modulatory effect of glycine and $L$-arginine on oxidative stress and necrosis induced by CDDP as well as on CDDP uptake by kidney. After $4 \mathrm{~h}$ of incubation with $2 \mathrm{mM}$ CDDP, nephrotoxicity was demonstrated by significant increased lactate dehydrogenase leakage, decreased ability of the slices to reduce 3-(4,5-dimethylthiazol-2yl)-2,5-diphenyltetrazolium bromide, increased lipid peroxides and depleted reduced glutathione. Also, CDDP significantly inhibited pyruvate-stimulated gluconeogenesis. Histopathological examination of RCS confirmed the occurrence of tubular coagulative necrosis in cortex and corticomedullary regions. Preincubation of RCS with $1 \mathrm{mM}$ glycine or L-arginine $1 \mathrm{~h}$ before CDDP addition significantly attenuated the oxidative stress and tubular necrotic effects of CDDP. L-Arginine showed greater antioxidant properties while glycine showed a greater antinecrotic effect. Moreover, the nephroprotective effect was mediated through lowering the platinum uptake by RCS. However, they could not counteract the inhibition of gluconeogenesis induced by CDDP. In conclusion, the present study sheds light on the mechanisms involved in glycine and $\mathrm{L}$-arginine nephroprotection.
\end{abstract}

Keywords: Cisplatin (CDDP), nephrotoxicity, glycine, L-arginine, renal cortical slices (RCS), lactate dehydrogenase (LDH)

\footnotetext{
*Address correspondence to:

Dr. Ebtehal El-Demerdash, Department of Pharmacology \& Toxicology, Faculty of Pharmacy, Ain Shams University, Abasia, Cairo, Egypt.

e-mail: ebtehal_dm@yahoo.com
}

\section{Introduction}

Cisplatin (cis-diamminedichloroplatinum; CDDP) is an effective chemotherapeutic agent and is successfully used in the treatment of a wide range of tumors (1). Its chief limit to greater efficacy, however, is its nephrotoxicity. Clinically, CDDP nephrotoxicity is often seen after 10 days of CDDP administration and is manifested as lower renal blood flow and glomerular filtration rate, high serum creatinine, and reduced serum magnesium and potassium levels (2). The mechanism by which CDDP produces renal injury is not well understood. It is probably related to its preferential uptake by the proximal tubular cells especially in the $\mathrm{S} 3$ segment (3). The renal microenvironmental changes following CDDP treatment is a complex process and could be broadly categorized into two distinct pathophysiological mechanisms. The first mechanism includes primary promoters of cellular damage such as inhibition of protein synthesis and depletion of glutathione and other protein-thiols while the secondary sequelae of established cell damage includes inactivation of transport proteins, lipid peroxidation and mitochondrial damage $(4,5)$. In addition, the changes in renal hemodynamics were also found to play an important role in CDDP-induced nephrotoxicity (6). CDDP toxicity in proximal tubules is characterized by tubular cell damage and subsequently, the injured renal tubules lose the ability to reabsorb water which lead to dehydration and loss of body weight (7).

Several strategies have emerged to ameliorate CDDP nephrotoxicity. Concomitant administration of chemoprotective agents becomes an important approach (8). For two decades, it has been proved that amino acids (9) and peptides derived from protein (10) possess vasodilatory effects on renal vessels and increase renal blood flow and glomerular filtration rate. Among nephroprotecive amino acids, glycine and $\mathrm{L}$-arginine are of great importance. It was reported that glycine exerted a cytoprotective effect in various forms of acute renal injury; it protected against hypoxic injury to rabbit renal proximal tubular cells (11), ouabaininduced injury in proximal tubule cells (12) and cyclosporine-induced cellular necrosis (13). Similarly, 
L-arginine, a nitric oxide $(\mathrm{NO})$ precursor, has been previously reported to have protective effects in drug induced renal injury where it was shown to attenuate renal dysfunction and oxidative stress in indomethacin (14), gentamicin (15), cyclosporin (16), and iron (17) induced renal impairment in rats.

Regarding CDDP nephrotoxicity, few studies tried to ameliorate its nephrotoxicity using either glycine $(18,19)$ or L-arginine $(20,21)$ and they reported a hemodynamic nephroprotective mechanism involving NO production. However, this hemodynamic mechanism may not be the sole one, since the nephroprotective effects were partially blocked by co-administration of the NO synthase inhibitor; $\mathrm{N}^{\mathrm{G}}$-nitro-L-arginine methyl ester (21). In addition, D-arginine, which is not a substrate for NO and has no vasodilating effect, is also reported to ameliorate the renal dysfunction that is induced by CDDP $(19,22)$. Thus, glycine and L-arginine might have nephroprotective effects against CDDP nephrotoxicity independent of their renal hemodynamic actions.

Therefore, the present study was designed to elucidate and compare the role of non hemodynamic mechanisms in protective actions afforded by both glycine and $\mathrm{L}$-arginine against CDDP-induced nephrotoxicity. We have investigated the possible modulatory effect of glycine and L-arginine on oxidative stress induced by CDDP as well as on CDDP uptake by kidney. We used a renal cortical slices (RCS) model because it is easily prepared, manipulated and contains populations of cells targeted by platinum anticancer agents; furthermore it is not influenced by uncontrollable hormonal, neurogenic and hemodynamic factors $(23,24)$.

\section{Materials and Methods}

\subsection{Chemicals}

CDDP was obtained from Merk Ltd., Cairo, Egypt. L-Arginine and glycine were obtained from Sigma-Aldrich, St Louis, MO, USA. Concentrations of the drugs used were selected as previously reported (25) as well as from pilot experimental trials of the present study. $n$-butanol, dimethyl sulfoxide (DMSO), 3-(4,5-dimethylthiazol-2yl)-2,5-diphenyltetrazolium bromide (MTT), 5,5'-dithiobis-2-nitrobenzoicacid (DTNB), ethylenediamine tetraacetic acid disodium salt (EDTA), D-glucose, $\mathrm{N}$-(2hydroxyethyl) piperazine- $N$ '-2-ethanesulfonic acid (HEPES), 1,1',3,3'-tetramethoxypropane, potassium dihydrogen phosphate, reduced glutathione (GSH), trichloroacetic acid (TCA), thiobarbituric acid (TBA), and Triton X-100 were purchased from Sigma-Aldrich. All these chemicals were dissolved in incubation medium prior to use. Other chemicals were analytical reagents.

\subsection{Animals}

Male Sprague Dawley rats were obtained from the Nile
Co. for Pharmaceuticals and Chemical industries, Cairo, Egypt. Animals were kept in the animal house of Faculty of Pharmacy, Ain Shams University, under suitable laboratory conditions on a standard diet and water ad libitum. The standard diet pellets were obtained from ElNasr Chemical Company, Cairo, Egypt. Animals were handled under a protocol of care of Animal Research Ethics Committee (Ain Shams University).

\subsection{Preparation and incubation of RCS}

RCS were prepared as described previously (25). After fasting overnight, the animal was killed by decapitation, then kidneys were rapidly removed, decapsulated and placed into ice-cold saline. The slices $(0.3-0.5 \mathrm{~mm}$ thickness) were prepared with a razor blade on a Petri plate in an ice-bath. The slices weighing 150-200 mg were loaded into an incubation flask with cap. Each flask contained $5 \mathrm{~mL}$ incubation medium $(134 \mathrm{mM} \mathrm{NaC}$, $5.9 \mathrm{mM} \mathrm{KCl}, 1.5 \mathrm{mM} \mathrm{CaCl}_{2}, 1.2 \mathrm{mM} \mathrm{MgCl} 2,11.5 \mathrm{mM}$ glucose, and $5.8 \mathrm{mM}$ HEPES buffer), gassed with pure oxygen. CDDP was added to the incubation medium to a final concentration of $2 \mathrm{mM}$. The medium for control slices was free of CDDP. The RCS were incubated at $37^{\circ} \mathrm{C}$ on a horizontal shaker (100 cycle/min).

\subsection{Experimental design}

In order to establish the optimal experimental conditions, a time course study of CDDP nephrotoxicity as well as a dose response study of glycine and L-arginine nephroprotection was carried out by assessing membrane integrity using a lactate dehydrogenase (LDH) leakage test. Then, based on data obtained from the preliminary study, the mechanisms of nephroprotection of glycine and L-arginine were studied using 6 groups of RCS.

\subsection{Time course study of CDDP-induced nephrotoxicity in rat RCS using LDH leakage method}

RCS were incubated with $2 \mathrm{mM}$ CDDP for different time intervals: $0.5,1,2,3,4$, and $6 \mathrm{~h}$. LDH leakage was assessed in the incubation medium according to the method described by Tietz (26) by measuring the rate of conversion of lactate to pyruvate and subsequent increase in the absorbance of nicotinamide adenine dinucleotide reduced form (NADH) formed at $340 \mathrm{~nm}$.

2.6. Determination of the protective concentrations of glycine and L-arginine against CDDP-induced $\mathrm{LDH}$ leakage

RCS were incubated for $1 \mathrm{~h}$ with either glycine or L-arginine at different concentrations: 1,2 and $4 \mathrm{mM}$. Then, $2 \mathrm{mM}$ CDDP was added and the slices were incubated for $4 \mathrm{~h}$ to choose the optimum concentration of amino acid to be used in further studies. 


\subsection{Assessment of protective mechanisms of glycine and L-arginine against CDDP-induced renal injury}

Rat RCS were classified into 6 groups (five sets/group); control, CDDP (2 mM), glycine (1 mM), L-arginine (1 $\mathrm{mM}), \mathrm{CDDP}+$ glycine, $\mathrm{CDDP}+\mathrm{L}$-arginine. Amino acids were added $1 \mathrm{~h}$ before CDDP.

\subsubsection{Assessment of CDDP-induced cytotoxicity in RCS}

CDDP-induced cytotoxicity in RCS was examined by MTT assay (27). Briefly, after $4 \mathrm{~h}$ of CDDP incubation, the slices were blotted with filter paper and incubated in $4 \mathrm{~mL}$ of a second incubation medium containing MTT $\left(1.25 \mathrm{mg} / \mathrm{mL} ; 3 \mathrm{mM}\right.$ final concentration) at $37^{\circ} \mathrm{C}$ for 30 $\mathrm{min}$. The slices were homogenized in $5 \mathrm{~mL}$ distilled water at 7,000 rpm for $1 \mathrm{~min}$ and centrifuged at 5,000 rpm for $5 \mathrm{~min}$ to remove particulate matter. An aliquot $(0.25 \mathrm{~mL})$ of the resulting supernatant of each group was added to a microcentrifuge tube and $0.5 \mathrm{~mL}$ DMSO was added to each tube followed by $0.5 \mathrm{~mL}$ glycine buffer $(\mathrm{pH} 10.5)$ containing $0.2 \mathrm{M}$ glycine and $0.1 \mathrm{M} \mathrm{NaCl}$. After mixing, the absorbances were measured at $570 \mathrm{~nm}$. The results were expressed as optical density $\mathrm{OD} / \mathrm{mg}$ wet tissue.

\subsubsection{Determination of lipid peroxides and GSH content in $R C S$}

After incubation, RCS were removed, rinsed, blotted, and homogenized with saline at a ratio of 1:10 (w/v). Lipid peroxides were assessed by measuring malonaldehyde content using TBA assays (28). Briefly, an aliquot of $0.5 \mathrm{~mL}$ of the homogenate was pipetted into a $10-\mathrm{mL}$ centrifuge tube followed by the addition of $2.5 \mathrm{~mL}$ of $20 \%$ TCA and $1 \mathrm{~mL}$ of $0.67 \%$ TBA. The mixture was heated for $15 \mathrm{~min}$ in a boiling water bath. After cooling, $4 \mathrm{~mL}$ of $n$-butanol was added and mixed vigorously. After centrifugation, the supernatant was read at $532 \mathrm{~nm}$ spectrophotometrically. Malonaldehyde concentration was calculated using a standard curve of 1,1,3,3-tetraethoxypropane and was expressed as nmol/g tissue. To determine GSH, $0.5 \mathrm{~mL}$ of homogenate was added to a tube with $0.5 \mathrm{~mL} \mathrm{10 \%} \mathrm{TCA.} \mathrm{The} \mathrm{tubes} \mathrm{were}$ gently shaken intermittently for $15 \mathrm{~min}$ followed by centrifugation at 2,000 rpm for $5 \mathrm{~min}$. A $0.2 \mathrm{~mL}$ aliquot of the resulting supernatant was added to a tube containing $1.7 \mathrm{~mL}$ phosphate buffer and $0.1 \mathrm{~mL} 5,5$ '-dithio-bis-(2nitrobenzoic acid), then mixed and read at $412 \mathrm{~nm}$ within $5 \min (29)$.

\subsubsection{Measurement of pyruvate stimulated gluconeo- genesis in RCS}

The slices were incubated in an incubation medium which contained $10 \mathrm{mM}$ pyruvate instead of glucose according to the study of Zhang et al. (25). After incubation, the slices were removed from the medium and blotted. The medium glucose content was spectrophotometrically determined using the glucose oxidase enzymatic assay (glucose assay kit) and expressed as $\mathrm{mg} / \mathrm{g}$ tissue.

\subsubsection{Determination of platinum content in RCS}

Platinum content in RCS was determined as described previously (30). After incubation of the slices, $1 \mathrm{~mL}$ of incubation medium of each sample was pipetted into a $10-\mathrm{mL}$ centrifuge tube, and the volume of each sample was brought to $1.1 \mathrm{~mL}$ with bidistilled water. The solutions were diluted with four equal volumes (4.4 $\mathrm{mL}$ ) of $1 \%$ Triton $\mathrm{X}-100$ solution. After vortex-mixing for $30 \mathrm{sec}, 20 \mu \mathrm{L}$ of the diluted sample was injected into the furnace using the autosampler. The apparatus used was a model 975 flamless atomic absorption spectrophotometer. A wavelength of $265.9 \mathrm{~nm}$ with a lamp current of $10 \mathrm{~mA}$ and spectral bandwidth of 0.2 $\mathrm{nm}$ was employed. Also, a background correction was utilized.

\subsubsection{Histopathological examination of RCS}

After incubation, RCS were fixed in $10 \%$ formol saline for $24 \mathrm{~h}$ and then washed in tap water. Serial dilutions of alcohol (methyl, ethyl and absolute ethyl) were used for dehydration. Specimens were cleared in xylene and embedded in paraffin at $56^{\circ} \mathrm{C}$ in a hot air oven for 24 h. Paraffin bees wax tissue blocks were prepared for sectioning at $4-\mu \mathrm{m}$ thickness using a slidge microtome. The tissue sections obtained were collected on glass slides, deparaffinized, and stained with hematoxylin and eosin stains for histopathological examinations with a light microscope.

\subsection{Statistical analysis}

Data are presented as mean \pm S.E.M. An unpaired $t$-test was used to compare two different treatment groups. Multiple comparisons for more than two treatment groups were carried out using one way analysis of variance (ANOVA) followed by the Tukey-Kramer test as post-hoc analysis. Statistical significance was acceptable at a level of $p<0.05$. Analysis of data was performed using GraphPad INSTAT. Tables and graphs were prepared using Prism software ver. 5 (GraphPad Software, San Diego, CA, USA).

\section{Results}

\subsection{Cytotoxicity markers}

First, CDDP-induced toxicity to the slices was determined by measuring LDH leakage from RCS. There was a time-dependent increase in LDH leakage and it was significantly increased as early as $1 \mathrm{~h}$ of 
CDDP incubation. After 4 and $6 \mathrm{~h}$ of incubation, LDH leakage was significantly increased by about 2 -fold as compared to the control value and therefore, the time interval $4 \mathrm{~h}$ was chosen for further studies (Figure 1). Preincubation of the slices with different concentrations $(1,2$, and $4 \mathrm{mM})$ of either glycine or L-arginine, $1 \mathrm{~h}$ before CDDP addition, nearly normalized the values of LDH leakage. The protective effect of glycine and L-arginine was not concentration-dependant as there is no significant difference between the effects of different concentrations of amino acids (Table 1). Therefore, the lowest concentration $(1 \mathrm{mM})$ was chosen for further studies.

Second, the MTT assay was used to assess cell damage as shown in Figure 2. After $4 \mathrm{~h}$, CDDP significantly decreased the ability of the slices to reduce MTT and the absorbance was decreased to $56 \%$ of the control value. On the other hand, the slices incubated with L-arginine alone showed a significant increase in the ability of the RCS to reduce MTT and the absorbance was increased to $121 \%$ of the control value.

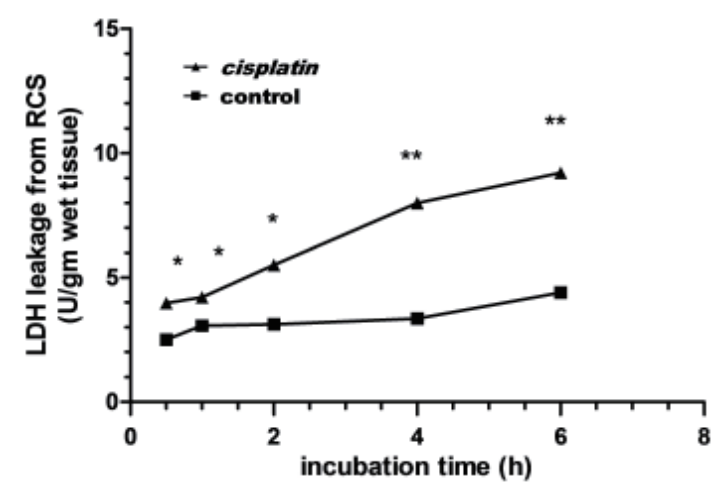

Figure 1. Time course study of the effect of $2 \mathrm{mM}$ CDDP on the release of LDH from rat RCS. Values given are the mean of 5 observations \pm S.E.M. ${ }^{*}$ Significantly different from the corresponding control at $p<0.05$ using unpaired student $t$-test. ${ }^{* *}$ Extremely significantly different from the corresponding control at $p<0.01$ using unpaired student $t$-test.

Table 1. Effect of glycine and L-arginine on CDDPinduced LDH enzyme leakage in the incubation medium of RCS

\begin{tabular}{lc}
\hline Treatments & $\begin{array}{c}\text { LDH leakage } \\
\text { (U/g wet weight) }\end{array}$ \\
\hline Control & $3.25 \pm 0.13$ \\
CDDP $(2 \mathrm{mM})$ & $6.45 \pm 0.50^{\mathrm{a}}$ \\
Glycine $(1 \mathrm{mM})+\mathrm{CDDP}$ & $3.66 \pm 0.24^{\mathrm{b}}$ \\
Glycine $(2 \mathrm{mM})+\mathrm{CDDP}$ & $4.28 \pm 0.22^{\mathrm{b}}$ \\
Glycine $(4 \mathrm{mM})+\mathrm{CDDP}$ & $4.06 \pm 0.25^{\mathrm{b}}$ \\
L-Arginine $(1 \mathrm{mM})+\mathrm{CDDP}$ & $3.17 \pm 0.29^{\mathrm{b}}$ \\
L-Arginine $(2 \mathrm{mM})+\mathrm{CDDP}$ & $2.74 \pm 0.19^{\mathrm{b}}$ \\
L-Arginine $(4 \mathrm{mM})+\mathrm{CDDP}$ & $3.23 \pm 0.21^{\mathrm{b}}$ \\
\hline
\end{tabular}

RCS were incubated at $37^{\circ} \mathrm{C}$ in medium containing CDDP $(2 \mathrm{mM})$ with or without different concentrations of glycine or L-arginine. Amino acids were added $1 \mathrm{~h}$ prior to CDDP. For the control group, the RCS were incubated in the medium without any drugs. LDH leakage was measured after $4 \mathrm{~h}$ of CDDP addition. Each value represents a mean of 5 sets of rat RCS \pm S.E.M. ${ }^{a}$ and ${ }^{b}$ are significantly different from control or CDDP group, respectively, at $p<0.05$ using ANOVA followed by Tukey-Kramer as a post Hoc test.
Incubation of RCS with glycine alone did not show any significant change in MTT reduction as compared to the control group. Pre-incubation of RCS with either glycine or L-arginine, $1 \mathrm{~h}$ before CDDP addition, significantly counteracted the decrease in MTT reduction induced by CDDP reaching approximately $70 \%$ of the control value.

\subsection{Oxidative stress markers}

RCS content of GSH for the control group was $5.5 \pm$ $0.07 \mu \mathrm{mol} / \mathrm{g}$ wet tissue (Table. 2). Incubation of RCS with either glycine or L-arginine alone revealed no significant change in GSH content as compared to the control group. On the other hand, incubation of RCS with CDDP alone for $4 \mathrm{~h}$ significantly depleted GSH content to $58 \%$ of the control value. Pre-incubation of the slices with $1 \mathrm{mM}$ glycine or L-arginine for $1 \mathrm{~h}$ before CDDP addition, significantly counteracted the effect of CDDP. Glycine and L-arginine increased the GSH content to about $76 \%$ and $90 \%$ of the control group, respectively (Table 2).

Regarding kidney lipid peroxides, the control level was $44.5 \pm 0.56 \mathrm{nmol} / \mathrm{g}$ wet tissue (Table 2 ). Incubation of RCS with CDDP significantly increased the level of lipid peroxides reaching $131 \%$ of the control group. Pre-incubation of RCS with either glycine or L-arginine significantly normalized the level of lipid peroxides to 110 and $108 \%$ of the control group, respectively (Table 2 ). The RCS incubated with either glycine or L-arginine alone revealed no significant change in lipid peroxides level compared to the control group.

Co-incubation of RCS with pyruvate, a substrate of gluconeogenesis, in the presence of CDDP

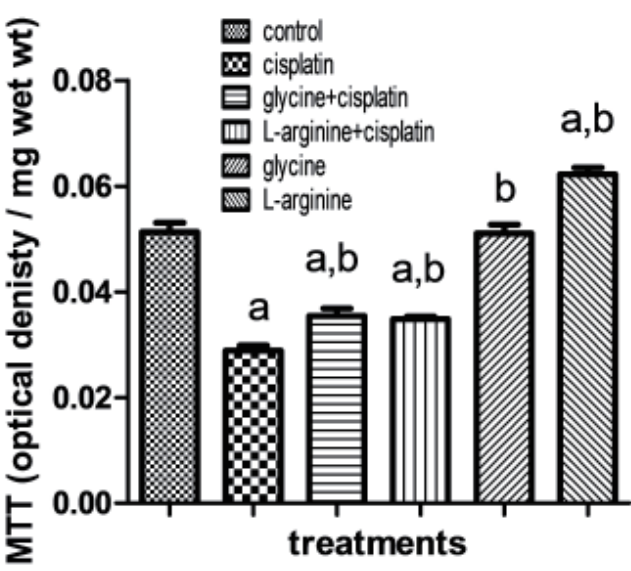

Figure 2. Effect of glycine or L-arginine on CDDP-induced cytotoxicity to RCS as percentage of control group using MTT assay. RCS were incubated at $37^{\circ} \mathrm{C}$ in medium containing CDDP $(2 \mathrm{mM})$ and/or glycine or L-arginine (1 $\mathrm{mM})$. Amino acids were added $1 \mathrm{~h}$ prior to CDDP. For control group, RCS were incubated in the medium without any drugs. MTT reduction was measured after $4 \mathrm{~h}$ of CDDP addition. Values given are the mean of 5 observations \pm S.E.M. a and b: Significantly different from control or CDDP group, respectively, at $p<0.05$ using ANOVA followed by TukeyKramer as a post Hoc test. 
Table 2. Effect of CDDP and/or glycine or L-arginine on lipid peroxides, GSH, and pyruvate-stimulated gluconeogenesis in RCS

\begin{tabular}{lccc}
\hline Treatments & Lipid peroxides (nmol/g wet tissue) & GSH ( $\mu$ mol/g wet tissue) & Glucose (mg/g wet tissue) \\
\hline Control & $44.5 \pm 0.6$ & $5.51 \pm 0.07$ & $7.81 \pm 0.50$ \\
CDDP & $58.4 \pm 1.2^{\mathrm{a}}$ & $3.22 \pm 0.06^{\mathrm{a}}$ & $2.64 \pm 0.18^{\mathrm{a}}$ \\
Glycine + CDDP & $48.9 \pm 1.9^{\mathrm{b}}$ & $4.16 \pm 0.06^{\mathrm{a}, \mathrm{b}}$ & $2.32 \pm 0.11^{\mathrm{a}}$ \\
L-Arginine + CDDP & $47.9 \pm 0.9^{\mathrm{b}}$ & $4.97 \pm 0.13^{\mathrm{a}, \mathrm{b}}$ & $1.92 \pm 0.13^{\mathrm{a}}$ \\
Glycine & $46.4 \pm 1.4^{\mathrm{b}}$ & $5.38 \pm 0.17^{\mathrm{b}}$ & $6.23 \pm 0.32^{\mathrm{b}}$ \\
L-Arginine & $45.8 \pm 1.2^{\mathrm{b}}$ & $5.67 \pm 0.13^{\mathrm{b}}$ & $6.44 \pm 0.36^{\mathrm{b}}$ \\
\hline
\end{tabular}

RCS were incubated at $37^{\circ} \mathrm{C}$ in medium containing CDDP $(2 \mathrm{mM})$ and/or glycine or L-arginine $(1 \mathrm{mM})$. Amino acids were added $1 \mathrm{~h}$ prior to CDDP. For the control group, RCS were incubated in the medium without any drugs. Lipid peroxides, GSH and glucose were measured after $4 \mathrm{~h}$ of CDDP addition. Each value represents the mean of 5 sets of rat RCS \pm S.E.M. ${ }^{a}$ and ${ }^{5}$ are significantly different from control or CDDP group, respectively, at $p<0.05$ using ANOVA followed by Tukey-Kramer as a post Hoc test.

showed a marked depression of pyruvate-stimulated gluconeogenesis to $34 \%$ of the control value (Table 2). On the other hand, neither glycine nor L-arginine had a significant effect on the gluconeogenic capacity of RCS. Addition of either glycine or L-arginine $1 \mathrm{~h}$ before CDDP did not show any protective effect on CDDP-induced inhibition of gluconeogenesis.

\subsection{CDDP uptake by renal cortical slices}

In order to know whether the studied amino acids influenced uptake of CDDP by RCS, CDDP concentration in the medium was determined and subtracted from the original amount of CDDP added to the medium. The platinum content in the RCS incubated with CDDP alone was $2.23 \pm 0.07 \mathrm{mg} / \mathrm{g}$ wet tissue. Preincubation of RCS with either glycine or L-arginine significantly decreased the kidney content of platinum to 86 and $91 \%$ compared to the CDDP group (Figure 3).

\subsection{Histopathological examination of RCS}

Histopathological examination of kidney specimens revealed normal renal glomeruli and tubules in the control group (Figures 4A and 4B). Also, RCS incubated with either glycine or L-arginine alone did not show any significant histopathological changes compared to the control group. On the other hand, RCS incubated with CDDP for $4 \mathrm{~h}$ showed tubular degeneration which varied according to kidney zone. Coagulative necrosis was observed in the tubular lining epithelium at the cortex and corticomedullary junction $(++++)$ (Figures $4 \mathrm{C}$ and 4D). Coagulative necrosis is an irreversible grade of necrotic cell death and tubular cells could not regenerate after removal of toxic insult (Table 3).

Pre-incubation of slices with glycine $1 \mathrm{~h}$ prior to CDDP addition ameliorated the histopathological changes induced by CDDP. Most of the renal tubules and glomeruli in the cortex were intact while other few tubules showed nuclear pyknosis at the cortex $(++)$ as well as the corticomedullary junction $(+)$ and medulla (Figures 4E and 4F). Pyknosis is the first stage in tubular degeneration, in which nuclei showed chromatin condensation without chromatolysis. Also, pre-incubation

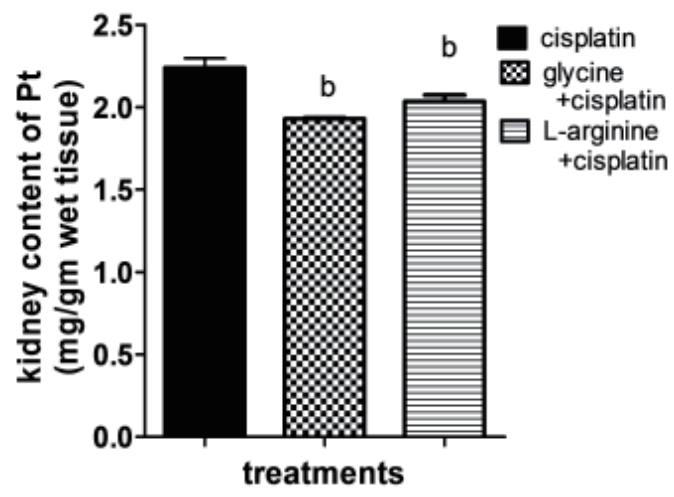

Figure 3. Effect of glycine or L-arginine on CDDP uptake by rat RCS expressed as kidney content of platinum. RCS were incubated at $37^{\circ} \mathrm{C}$ in medium containing CDDP $(2$ $\mathrm{mM})$ and/or glycine or L-arginine $(1 \mathrm{mM})$. Amino acids were added $1 \mathrm{~h}$ prior to CDDP. Platinum content was measured after $4 \mathrm{~h}$ of CDDP addition. Values given are the mean of 5 observations \pm S.E.M. b: significantly different from CDDP group at $p<0.05$ using ANOVA followed by Tukey-Kramer as a post Hoc test.

of slices with L-arginine $1 \mathrm{~h}$ prior to CDDP addition showed nephroprotective and antinecrotic effects but to a less degree as compared to the glycine effect (Table 3; Figures $4 \mathrm{G}$ and $4 \mathrm{H}$ ).

\section{Discussion}

The present study was designed to elucidate and compare the role of non hemodynamic mechanisms in protective actions afforded by glycine and L-arginine against CDDP-induced nephrotoxicity. The first point discussed was assessment of CDDP nephrotoxicity using LDH leakage; a sensitive indicator for cell damage (23). It was found that both glycine and L-arginine significantly protected against CDDPinduced LDH leakage and loss of cell viability as measured by the MTT assay. These findings add further evidence for the potential cytoprotective effects of these amino acids. Since MTT is mainly reduced by the mitochondrial dehydrogenases (27), enzymes that were inhibited directly by CDDP after incubation with RCS (31), our findings give evidence that both amino acids could ameliorate CDDP-induced mitochondrial toxicity. 

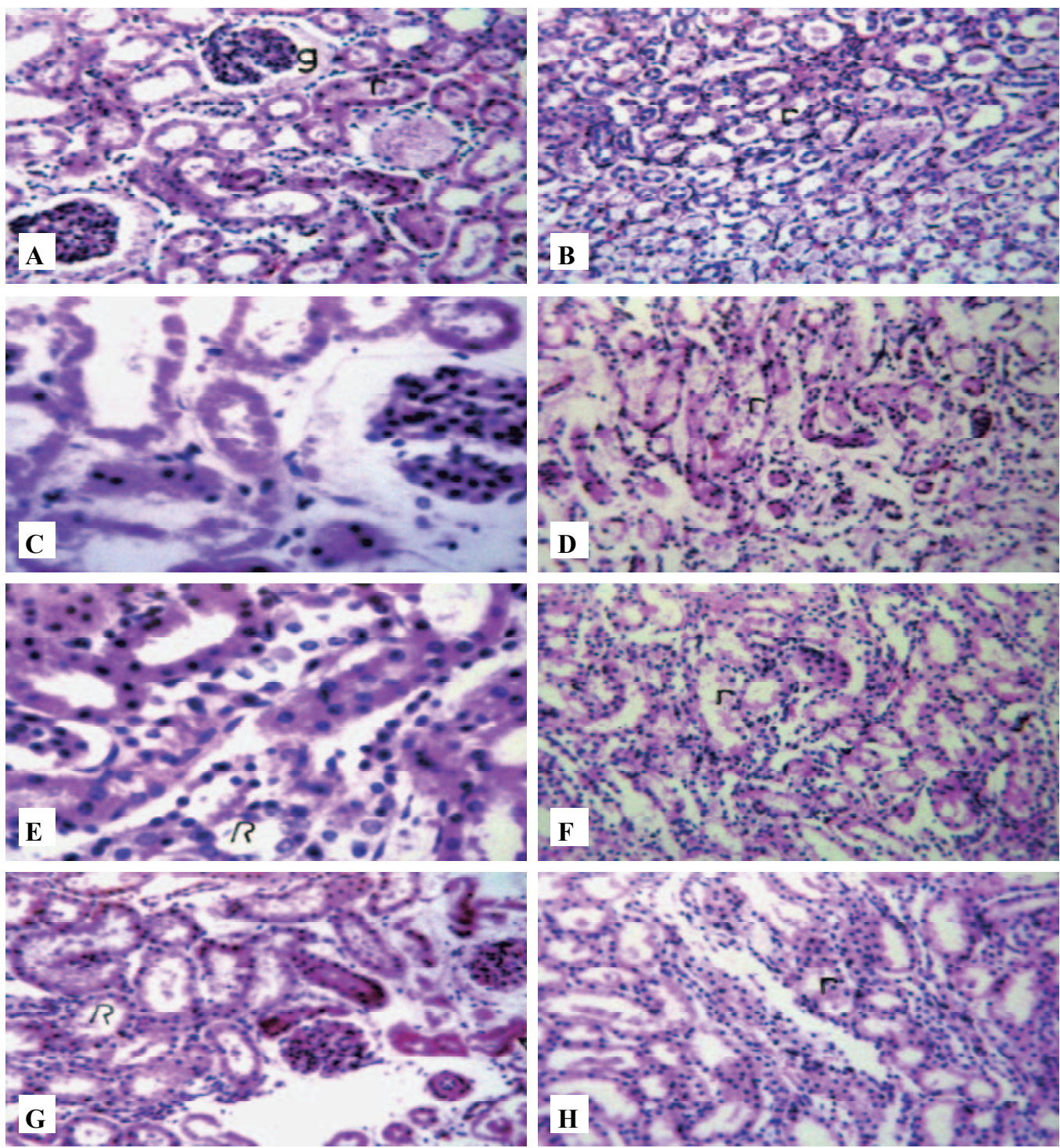

Figure 4. Photomicrographs of sections in the kidneys stained with hematoxylin-eosin stain. A and B: Sections taken from RCS of control group and show normal glomeruli $(\mathrm{g})$ and tubules $(\mathrm{r})$ at the cortex portion (A), and normal renal tubules (r) at corticomedulary portion (B). Original magnification, $\times 64$. C and D: Sections taken from RCS of CDDP group and show coagulative necrosis in the tubular lining epithelium $(r)$ of the cortical region graded as $++++(\mathbf{C})$, and also the corticomedular region graded as ++++ (D). Original magnification, $\times 160$ and $\times 64$ for $\mathbf{C}$ and $\mathbf{D}$, respectively. $\mathbf{E}$ and $\mathbf{F}$ : Sections taken from RCS of glycine + CDDP group and show most of the renal tubules and glomeruli in the cortex were intact with mild degenerative changes while a few other tubules showed nuclear pyknosis and complete absence of their nuclei with intact basement membrane graded as ++ at the cortex $(\mathbf{E})$ and as + at corticomedulary junction $(\mathbf{F})$. Original magnification, $\times 160$ and $\times 64$ for $\mathbf{E}$ and $\mathbf{F}$, respectively. $\mathbf{G}$ and $\mathbf{H}$ : Sections taken from RCS of L-arginine + CDDP group and show intact glomeruli with coagulative necrosis in multiple numbers of renal tubules and other tubules show complete loss of the lining epithelium while the basement membrane is still intact $(\mathbf{G})$ and pyknosis of the nuclei of the lining epithelial cells at the corticomedulary junction graded as $+(\mathbf{H})$. Original magnification, $\times 64$

Table 3. Histopathological alterations in RCS

\begin{tabular}{|c|c|c|c|c|c|c|c|c|c|c|c|c|c|c|c|c|c|c|}
\hline \multirow{2}{*}{ Histopathological alterations } & \multicolumn{3}{|c|}{ Control group } & \multicolumn{3}{|c|}{ CDDP group } & \multicolumn{3}{|c|}{ Glycine + CDDP } & \multicolumn{3}{|c|}{ L-Arginine + CDDP } & \multicolumn{3}{|c|}{ Glycine } & \multicolumn{3}{|c|}{ L-Arginine } \\
\hline & $\mathrm{C}$ & $\mathrm{CM}$ & $\mathrm{M}$ & $\mathrm{C}$ & $\mathrm{CM}$ & M & $\mathrm{C}$ & $\mathrm{CM}$ & M & $\mathrm{C}$ & $\mathrm{CM}$ & M & $\mathrm{C}$ & $\mathrm{CM}$ & M & $\mathrm{C}$ & $\mathrm{CM}$ & M \\
\hline$\uparrow$ 1-Coagulative necrosis & - & - & - & ++++ & ++++ & - & - & - & - & ++++ & - & - & - & - & - & - & - & - \\
\hline \begin{tabular}{l|l} 
& 2-Pyknosis of the nuclei \\
& of the tubules
\end{tabular} & - & - & - & - & - & - & ++ & + & + & - & + & - & - & - & - & - & - & - \\
\hline \begin{tabular}{l|l}
$\mathcal{E}^{E}$ & 3-Degeneration of the \\
0 & 1 \\
$\delta$ & tubular lining epithellium
\end{tabular} & - & - & - & - & - & - & - & - & ++ & - & - & + & - & - & - & - & - & - \\
\hline \begin{tabular}{l|l}
$\delta_{0}$ & 4-Atrophic glomeruli \\
&
\end{tabular} & - & - & - & - & - & - & - & - & - & - & - & - & - & - & - & - & - & - \\
\hline
\end{tabular}

C, cortex; CM, corticomedullary portion; M, medulla. 
It is well known that the cellular response toward CDDP induced damage could lead to cell death either by apoptosis or necrosis. The type of cellular injury induced by CDDP depends on the extent of damage to proteins or molecules involved in the apoptotic pathway (32). To clarify the protective role of glycine and L-arginine in CDDP induced cell death, histopathological examination was performed and revealed that CDDP produced marked coagulative necrosis in the tubular epithelium of the cortex and corticomedullary region and these results are in accordance with previous studies $(24,33)$. Furthermore, histopathological examination did not reveal apoptosis or apoptotic bodies, a finding that may indicate sever cellular damage of proteins or molecules involved in the apoptotic pathway resulting in unfinished apoptosis or necrosis $(32,34)$. Regarding amino acids, glycine was found to be more effective than L-arginine in protection against CDDP induced necrosis at the cortex as glycine was capable of converting CDDP induced damage from coagulative necrosis to tubular degeneration and the first stage of nuclear necrosis; pyknosis. At the tubular degeneration stage, the kidney can regenerate its normal structure after CDDP treatment stoppage. In contrast, both of these amino acids have the same effect in the corticomedullary region.

Despite the fact that the underlying mechanism of CDDP-induced nephrotoxicity is still not clear, the avidity of platinum for sulphur containing ligands has been proposed in the hypothetical mechanisms explaining the nephrotoxicity of CDDP. The depletion of mitochondrial GSH is considered as an early and critical event during CDDP-induced lipid peroxidation and subsequent toxicity $(25,35)$. In the present study, it was found that, CDDP induced a significant depletion of GSH concomitant with a significant elevation of lipid peroxides, which is in accordance with previous invitro studies $(31,36)$; these findings add further evidence for the role of oxidative stress in CDDP-induced renal toxicity. Pre-incubation of RCS with either glycine or L-arginine significantly counteracted the effect of CDDP and restored the normal level of both GSH and lipid peroxides. The antioxidant properties of glycine $(37,38)$ and L-arginine $(21,39)$ had been previously reported in several oxidative stress situations. Although, glycine is a direct precursor of GSH, L-arginine showed a significant protective effect on GSH as compared to glycine effect. In an experiment with hypoxic proximal tubular cells, Weinberg and co-workers (40) observed that cytoprotection provided by glycine was accompanied by a preservation of cellular GSH and ATP levels. However, the depletion of GSH content or ATP levels of renal tubular cells did not reduce the cytoprotective activity of glycine. Therefore, they suggested that glycine might have a direct cytoprotective effect.

Besides the oxidative stress markers; pyruvatestimulated gluconeogenesis was measured in RCS.
Co-incubation of RCS with pyruvate, a substrate of gluconeogenesis, in the presence of CDDP showed marked depression of pyruvate-stimulated gluconeogenesis which reached $32 \%$ of the control value and theses results are in accordance with other previous studies performed in vitro (25). Addition of either glycine or L-arginine $1 \mathrm{~h}$ before CDDP did not show any protective effect on CDDP-induced inhibition of gluconeogenesis. Although glycine and L-arginine are gluconeogenic amino acids in kidney metabolism leading to a precursor for gluconeogenesis $(41,42)$, in the present study, they did not show any protective effect against CDDP induced inhibition of gluconeogenesis. These findings may indicate the existence of different mechanisms of CDDP induced nephrotoxicity beside free radical formation and oxidative stress. Indeed, previous studies are controversial concerning the role of oxidative stress in gluconeogensis and whether we can use the inhibition of gluconeogensis as a marker of oxidative stress or not. Zhang et al. (25) proposed that GSH depletion might be responsible for inhibition of the activities of the relevant gluconeogenic enzymes, e.g. phosphoenolpyruvate carboxykinase, glycerol-3phosphate dehydrogenase, and glucose-6-phosphatase. On the other hand, Hannmann and Baumann in two serial studies $(43,44)$, proposed that direct inhibition of the gluconeogenic enzyme glucose-6-phosphatase by CDDP or its metabolites might play a role in inducing the decrease of gluconeogenesis away from GSH depletion and oxidative stress effects of CDDP.

Finally, we tried to answer the question of whether nephroprotection can be achieved by inhibiting CDDP uptake by RCS in the presence of amino acids, either glycine or L-arginine. Since several investigators have observed a correlation between platinum levels and in-vitro toxicity (45), it is believed that the targeted inhibition of CDDP accumulation in renal cells could lead to a new therapeutic approach to preventing CDDP nephrotoxicity. In the present study, CDDP concentration in the medium was determined and subtracted from the original amount of CDDP added to the medium. The platinum content in the RCS incubated with CDDP alone was $2.23 \pm 0.07 \mathrm{mg} / \mathrm{g}$ wet tissue. Our results are in agreement with Zhang et al. (25) and Saleh et al. (46). Preincubation of RCS with glycine or L-arginine 1 $\mathrm{h}$ before CDDP addition showed a significant decrease in the kidney content of platinum to be 86 and $91 \%$, respectively, as compared to the CDDP group, a finding that reflects the possible inhibition of CDDP uptake in the putative mechanisms of nephroprotection afforded by both glycine and L-arginine.

Indeed, transporter mediated uptake is likely the major pathway in renal cells. The organic cation transporter (OCT 2) is the critical transporter for CDDP uptake in proximal tubules in both animals and humans (47). They mediate the basolateral-to-apical transport of 
several cationic compounds in renal tubular cells (48). On the basis of active transport of CDDP, cimetidine, an inhibitor of OCTs, could prevent CDDP-induced proximal tubule cell cytotoxicity or apoptosis (49). One study has been conducted on glycine, Heymen et al. (50) reported that the infusion of glycine into the rats $15 \mathrm{~min}$ before CDDP injection attenuated the early accumulation of platinum in the kidney, and may explain the protective effect of glycine. The results of this study confirmed the role of a hemodynamic effect of glycine, possibly through increasing renal blood flow and glomerular filtration rate and so accelerating CDDP excretion. Although, glycine is also actively transported by renal cells (51), it is not known whether it utilizes the OCT or not. As we mentioned before, RCS is a good model for studying CDDP nephrotoxicity because it contains populations of cells targeted by platinum anticancer agents and is not influenced by uncontrollable hormonal, neurogenic and hemodynamic factors. Therefore, the present study demonstrated that the decrease in CDDP uptake could be a direct effect of glycine on CDDP transportation. Further studies are warranted to explore whether glycine can affect the OCT carrier. On the other hand, in reviewing the literature, nothing concerning the effect of L-arginine on CDDP uptake by kidney has been reported. Thus, the findings of the present study add another new mechanism for L-arginine nephroprotection by reducing CDDP uptake. It is important to mention that L-arginine uptake was proven to be mediated through the cationic amino acid transporter (52) and so, it might interfere with CDDP uptake by the cationic transporters.

In conclusion, the present study clearly demonstrates that glycine and L-arginine could be promising drugs as nephroprotectors against CDDP-induced nephrotoxicity. Furthermore, this study sheds light on the mechanisms involved in their nephroprotection. They guard against oxidative stress induced by CDDP by restoring cellular defense mechanisms, replenishing kidney GSH content and normalizing lipid peroxidation. Also, the nephroprotective effect was mediated through lowering platinum uptake by the kidney tissue. Furthermore, they could ameliorate necrosis or renal tubule damage induced by CDDP. L-Arginine showed greater antioxidant properties while glycine showed greater antinecrotic effects. Further investigations are clearly warranted to establish the clinical applicability of these amino acids in patients with nephrotoxicity associated with CDDP administration and to explore possible interference of the antitumor activity of CDDP.

\section{Acknowledgement}

We thank Professor Adel B. Kholoussy (Department of Pathology, Faculty of Veterinary Medicine, Cairo University) for his kind help in the pathology part of this work.

\section{References}

1. Rosenberg B. Platinum complexes for the treatment of cancer: Why the search goes on. In: Ciplatin. Chemistry and biochemistry of a leading anticancer drug (Lippert B, ed.). Wiley-VCH, Basel, Switzerland. 1999; pp. 3-27.

2. Arany I, Safirstein RL. Cisplatin nephrotoxicity. Semin Nephrol. 2003; 23:460-464.

3. Andersson A, Fagerberg J, Lewensohn R, Ehrsson H. Pharmacokinetics of cisplatin and its monohydrated complex in humans. J Pharm Sci. 1996; 85:824-827.

4. Lau AH. Apoptosis induced by cisplatin nephrotoxic injury. Kidney Int. 1999; 56:1295-1298.

5. Kuhlmann MK, Burkhardt G, Köhler H. Insights into potential cellular mechanisms of cisplatin nephrotoxicity and their clinical application. Nephrol Dial Transplant. 1997; 12:2478-2480.

6. Winston JA, Safirstein R. Reduced renal blood flow in early cisplatin-induced acute renal failure in the rat. Am J Physiol. 1985; 249:F490-F496.

7. Ali BH, Al-Moundhri M, Tageldin M, Al Husseini IS, Mansour MA, Nemmar A, Tanira MO. Ontogenic aspects of cisplatin-induced nephrotoxicity in rats. Food Chem Toxicol. 2008; 46:3355-3359.

8. Hospers GA, Eisenhauer EA, de Vries EG. The sulfhydryl containing compounds WR-2721 and glutathione as radioand chemoprotective agents. A review, indications for use and prospects. Br J Cancer. 1999; 80:629-638.

9. Brezis M, Silva P, Epstein FH. Amino acids induce renal vasodilatation in isolated perfused kidney: Coupling to oxidative metabolism. Am J Physiol. 1984; 247 : H999-H1004.

10. Rodríguez-Iturbe B, Herrera J, García R. Relationship between glomerular filtration rate and renal blood flow at different levels of protein-induced hyperfiltration in man. Clin Sci (Lond). 1988; 74:11-15.

11. Weinberg JM, Davis JA, Abarzua M, Rajan T. Cytoprotective effects of glycine and glutathione against hypoxic injury to renal tubules. J Clin Invest. 1987; 80:1446-1454.

12. Weinberg JM, Davis JA, Abarzua M, Smith RK, Kunkel R. Ouabain-induced lethal proximal tubule cell injury is prevented by glycine. Am J Physiol. 1990; 258:F346-F355.

13. Thurman RG, Zhong Z, von Frankenberg M, Stachlewitz RF, Bunzendahl H. Prevention of cyclosporin-induced nephrotoxicity with dietary glycine. Transplantation. 1997; 63:1661-1667.

14. Basivireddya J, Jacob M, Pulimooda AB, Balasubramanian KA. Indomethacin-induced renal damage: Role of oxygen free radicals. Biochem Pharmacol. 2004; 67:587-599.

15. Secilmia MA, Karatas Y, Yorulmaza O, Buyukafşar K, Singirik E, Doran F, Inal TC, Dikmen A. Protective effect of L-arginine intake on the impaired renal vascular responses in the Gentamicin-treated rats. Nephron Physiol. 2005; 100:13-20.

16. Kuruş M, Eşrefoğlu M, Bay A, Oztürk F. Protective effect of oral L-arginine supplementation on cyclosporine induced nephropathy in rats. Int Urol Nephrol. 2005; 37:587-594.

17. Gupta A, Chander V, Sharma S, Chopra K. Sodium nitroprusside and L-arginine attenuates ferric nitrilotriacetate-induced oxidative renal injury in rats. Toxicology. 2007; 232:183-191.

18. Heyman SN, Rosen S, Silva P, Spokes K, Egorin MJ, Epstein FH. Protective action of glycine in cisplatin 
nephrotoxicity. Kidney Int. 1991; 40:273-279.

19. Li Q, Bowmer CJ, Yates MS. The protective effect of glycine in cisplatin nephrotoxicity: Inhibition with NGnitro-L-arginine methyl ester. J Pharm Pharmacol. 1994; 46:346-351.

20. Cernadas MR, López-Farré A, Riesco A, Gallego MJ, Espinosa G, Digiuni E, Hernando L, Casado S, Caramelo C. Renal and systemic effect of amino acids administered separately: Comparison between L-arginine and nonnitric oxide donor amino acids. J Pharm Exp Ther. 1992; 263:1023-1029.

21. Saleh S, El-Demerdash E. Protective effects of L-arginine against cisplatin-induced renal oxidative stress and toxicity: Role of nitric oxide. Basic Clin Pharmacol Toxicol. 2005; 97:91-97.

22. Li Q, Bowmer C, Yates MS. Effect of arginine on cisplatin-induced acute renal failure in rat. Biochem Pharmacol. 1994; 47:2298-2301.

23. Smith JH. The use of renal cortical slices from the Fischer 344 rat as an in-vitro model to evaluate nephrotoxicity. Fundam Appl Toxicol. 1988; 132-142.

24. Vickers AE, Rose K, Fisher R, Saulnier M, Sahota P, Bentley P. Kidney slices of human and rat to characterize cisplatin-induced injury on cellular pathways and morphology. Toxicologic Pathol. 2004; 32:577-590.

25. Zhang JG, Zhong LF, Zhang M, Xia YX. Protection effects of procaine on oxidative stress and toxicities of renal cortical slices from rats caused by cisplatin in vitro. Arch Toxicol. 1992; 66:354-358.

26. Tietz NW. Clinical guide to laboratory tests. 3rd ed., W.B. Saunders Co., Philadelphia, PA, 1995.

27. Mosmann T. Rapid colorimetric assay for cellular growth and survival: Application to proliferation and cytotoxicity assays. J Immunol Methods. 1983; 65:55-63.

28. Buege JA, Aust SD. Microsomal lipid peroxidation. Methods Enzymol. 1978; 52:302-310.

29. Ellman GL. Tissue sulfhydryl groups. Arch Biochem Biophys. 1959; 82:70-77.

30. el-yazigi A, Al-Saleh I. Rapid determination of platinum by flameless atomic absorption spectrophotometry following the administration of cisplatin to cancer patients. Ther Drug Monit. 1986; 8:318-320.

31. Zhang JG, Lindup WE. Role of mitochondria in cisplatin-induced oxidative damage exhibited by rat renal cortical slices. Biochem Pharmacol. 1993; 45:2215-2222.

32. Gonzalez VM, Fuertes MA, Alonso C, Perez JM. Is Cisplatin-induced cell death always produced by apoptosis? Mol Pharmacol. 2001; 59:657-663.

33. Cristofori P, Zanetti E, Fregona D, Piaia A, Trevisan A. Renal proximal tubule segment-specific nephrotoxicity: An overview on biomarkers and histopathology. Toxicologic Pathol. 2007;35:270-275.

34. Delmastro DA, Li J, Vaisman A, Solle M, Chaney SG. DNA damage inducible-gene expression following platinum treatment in human ovarian carcinoma cell lines. Cancer Chemother Pharmaco. 1997; 39:245-253.

35. Mora Lde O, Antunes LM, Francescato HD, Bianchi Mde L. The effects of oral glutamine on cisplatin-induced nephrotoxicity in rats. Pharmacol Res. 2003; 47:517-522.

36. Yoshida M, Itzuka K, Hara M, Nishijima H, Shimada A, Nakada K, Satoh Y, Akama Y, Terada A. Prevention of nephrotoxicity of cisplatin by repeated oral administration of ebslenin rats. Tohoku J Exp Med. 2000; 191:209-220.

37. Sobage K, Roeser NF, Venkatachalam MA, Weinberg JM. Differential cytoprotection by glycine against oxidant damage to proximal tubule cells. Kidney Int. 1996; 50:845-854.

38. Shaikh ZA, Tang W. Protection against chronic cadmium toxicity by glycine. Toxicology. 1999; 132:139-146.

39. Mansour M, Daba MH, Gado A, Al-Rikabi A, Al-Majed A. Protective effect of $\mathrm{L}$-arginine against nephrotoxicity induced by cyclosporine in normal rats. Pharmacol Res. 2002; 45:441-446.

40. Weinberg JM, Davis JA, Abarzua M, Kiani T. Relationship between cell adenosine triphosphate and glutathione content and protection by glycine against hypoxic proximal tubule cell injury. J Lab Clin Med. 1989; 113:612-622.

41. Krebs HA, Yoshida T. The gluconeogenic capacity of the kidney cortex of various species. Biochem J. 1963; 89:398-400.

42. Hetenyi G Jr, Anderson PJ, Raman M, Ferrarotto C. Gluconeogenesis from glycine and serine in fasted normal and diabetic rats. Biochem J. 1988; 253:27-32.

43. Hannemann J, Baumann K. Nephrotoxicity of cisplatin, carboplatin and transplatin, a comparative in vitro study. Arch Toxicol. 1990; 64:393-400.

44. Hannemann J, Baumann K. Cisplatin-induced lipid peroxidation and decrease of gluconeogenesis in rat kidney cortex: Different effects of antioxidants and radical scavengers. Toxicology. 1988; 51:119-132.

45. Yao X, Panichpisal K, Kurtzman N, Nugent K. Cisplatin Nephrotoxicity: A review. Am J Med Sci. 2007; 334:115-124.

46. Saleh S, Ain-Shoka AA, El-Demerdash E, Khalef MM. Prevention of cisplatin-induced nephrotoxicity by an angiotensin-II receptor blocker, losratan. Chemotherapy. 2009; 55:399-406

47. Kröning R, Lichtenstein AK, Nagami GT. Sulfurcontaining amino acids decrease cisplatin cytotoxicity and uptake inrenal tubule epithelial cell lines. Cancer Chemother Pharmacol. 2000; 45:43-49.

48. Pabla N, Dong Z. Cisplatin nephrotoxicity: Mechanisms and renoprotective strategies. Kidney Int. 2008; 73:994-1007.

49. Ludwig T, Riethmüller C, Gekle M, Schwerdt G, Oberleithner H. Nephrotoxicity of platinum complexes is related to basolateral organic cation transport. Kidney Int. 2004; 66:196-202.

50. Heymen SN, Spokes K, Egorin MJ, Epstein FH. Glycine reduces early renal parenchymal uptake of cisplatin. Kidney Int. 1993; 43:1226-1228.

51. Scallera V, Corcelli A, Frassanito A, Storelli C. Chloride dependence of the sodium-dependent glycine transport in pig kidney cortex brush border membrane vesicles. Biochim Biophys Acta. 1987; 903:1-10.

52. Kim JW, Closs EI, Albritton LM, Cunningham JM. Transport of cationic amino acids by the mouse ecotropic retrovirus receptor. Nature. 1991; 352:725-728.

(Received October 19, 2010; Revised November 27, 2010; Accepted December 6, 2010) 